\title{
Root resection and PRF as an alternative treatment option: A case report
}

\author{
Parul Chhabra $^{1, *}$, Arvind Garg ${ }^{2}$, Pujit Chhabra ${ }^{3}$, Deepak Chhabra ${ }^{4}$ \\ ${ }^{1}$ Periodontist, ${ }^{2} \mathrm{HOD},{ }^{3}$ Post Graduate Student Orthodontist, ${ }^{4}$ Pharmacist, ${ }^{\mathbf{4}}$ Kurukshetra University, ${ }^{\mathbf{1 - 3} J a n}$ Nayak Ch. Devi Lal \\ Dental College, Sirsa, Haryana, India
}

*Corresponding Author:

Email: drparulc@gmail.com

\begin{abstract}
Periodontal and endodontic tissue structures are closely related odontogenic, which promotes spread of infection. But the desire of patients to maintain their dentition enforce and encourage the dentist to conserve the tooth. In past tooth with severe bone loss and endodontically compromised, resective surgery was employed but today regenerative therapy has potential to restore lost structure esthetically and functionally. In the clinical case presented in this paper, there was bone loss on the site and successful healing was obtained by doing endodontic treatment and using PRF.
\end{abstract}

Keywords: PRF, Root resection, Furcation.

\section{Introduction}

A 40 year old male patient reported to Periodontics OPD at JCD dental College, Sirsa with chief complaint of food lodgment in the upper left back region. On Intraoral examination, gingiva around 26 was red and recession was present. (Fig. 1) On radiographic examination, Intraoral periapical radiograph revealed Root canal treatment was done in 26 but bone loss was present with a distobuccal root of maxillary first molar with furcation involvement, mesiobuccal and palatal root showed adequate bone support so it was decided to retain these roots and resect distobuccal root. (Fig. 2) Phase I therapy was completed and tissue was examined on subsequent visits. After achieving appropriate local anesthesia, full thickness partial thickness flaps were elevated on the buccal and palatal aspect for proper access, visualization and instrumentation. (Fig. 3) thorough debridement was done of affected area and a small amount of facial bone was removed to provide access for elevation and facilitate root removal followed by horizontal resection of distobuccal root with high-speed tapered fissure carbide bur just apical to cementoenamel junction of the tooth. The placement of a curved periodontal probe into or through the furcation aids in orienting the angle of the resection. After sectioning, the root was elevated from its socket. (Fig. 4) Care was taken not to traumatize bone on the remaining roots or to damage an adjacent tooth. Removal of the root provided visibility to the furcation aspect of the remaining roots and simplified the debridement of the furcation. Furcation area was trimmed to and socket was irrigated with saline. PRF was prepared by protocol given by Choukroun et al. $10 \mathrm{ml}$ of venous blood was collected in dry glass tubes and centrifuged at 3000 RPM for 10 minutes (Process protocol, Nice, France). The clot was picked up with forceps, and the red thrombus (the fraction of red blood cells) was eliminated with scissors. PRF membrane was obtained by pressing the clot between two gauzes thereby squeezing out the fluids in the fibr in clot (Raja and Naidu 2008). Condensation of bone graft (Perioglass) was done in socket and as a membrane for guided bone regeneration, PRF dense matrix architecture covers, protect and stabilizes the Bone graft and operative site in general. (Fig. 5) Flaps were approximated \& sutured, COE pack was given. (Fig. 6) The occlusion was evaluated to facilitate healing. Two weeks after the surgery patient was recalled and sutures were removed. The patient was periodically recalled and evaluated. (Fig. 7)

\section{Discussion}

The treatment option of multirooted tooth with furcation involvement varies between non-surgical approaches consisting of scaling and root planning to surgical approaches consisting of odontoplasty, root resection, hemi section, regeneration \& bone grafting, tunnel preparation, final extraction depending on various factors. ${ }^{1}$ Root resection has been performed in dentistry since $1800 \mathrm{s.}^{2}$ According to literature, there are diverse opinions on the effectiveness of root resection therapy. It is reported by most of studies, even after five years the failure rate of root resection is low. Buhler (1988) reported that the failure rate of the tooth treated by root resection, over a seven-year of period, was $11 \%$.

Root resection is very technique sensitive and complex, so proper case selection is essential. ${ }^{3}$ the successful root resection therapy requires careful multidisciplinary approach, including endodontic treatment, periodontal surgery and prosthetic rehabilitation. ${ }^{1}$ Root separation or resection has been used successfully to retain teeth with furcation involvement. However, there are few disadvantages associated with it. As with any surgical procedure, it can cause pain and anxiety. Root surfaces that are reshaped by grinding in the furcation or at the site of hemi section are more susceptible to caries of ten a favorable result may be negated by decay after

IP International Journal of Periodontology and Implantology, October-December, 2018;3(4):128-130 $\quad 128$ 
treatment. Failure of endodontic therapy due to any reason will cause failure of the procedure. In addition, when the tooth has lost part of its root support, it will require a restoration to permit it to function independently or to serve as an abutment for a splint or a bridge. Unfortunately, a restoration can contribute to periodontal destruction, if the margins are defective or if non-occlusal surfaces do not have physiologic form. Also, an improperly shaped occlusal contact area may convert acceptable forces into destructive forces and predispose the tooth to trauma from occlusion and ultimate failure of root separation and resection. ${ }^{4}$

The distobuccal root of maxillary molar is the shortest root and has comparatively large root trunk. Thus distobuccal root is, therefore, often removed as part of root resection in maxillary molars. ${ }^{5,6}$ Root resection can be performed on vital or endodontically treated teeth. It is preferable, however to have pulp extirpation before the resection. This facilitates the performance of endodontic obturation, allows the endodontist to determine whether the canals can be adequately instrumented and also ensures the clinical feasibility of the procedure. If this not possible, then patency of canals determined, and the pulp chamber medicated before resection therefore, contamination of the pulp chamber by oral fluids does not occur. In addition, performing endodontic treatment before root resection may minimize the potential for postoperative pain. $^{7}$

Used as a covering membrane, PRF accelerates healing and closure of wound margins, stabilizes the graft materials and protects the surgical site from external aggressions. Mixed with graft material, PRF will serve as the biological cement between the particles and enhance neoangiogenesis and bone regeneration, particularly in stimulating osteoblastic proliferation and differentiation. ${ }^{8}$

The removal of a root alters the distribution of occlusal forces on the remaining roots. Therefore, it is wise to evaluate the occlusion of teeth from which roots have been resected and, if necessary, adjust the occlusion. Centric holds should be maintained, but eccentric forces should be eliminated from the area over the root that was removed. ${ }^{9}$

\section{Conclusion}

For molars with furcation involvement rootresection therapy is still a valid treatment option. The successful root resection requires careful multidisciplinary approach including endodontic treatment, periodontal treatment and prosthetic rehabilitation. Although long term prognosis and efficacy of the procedure is controversial, 1989 world workshop stated that "root resection is a procedure which still remains as a part of periodontal armamentarium to treat very specific cases which cannot be solved by any other therapeutic approach and where tooth in question has a high strategic value".

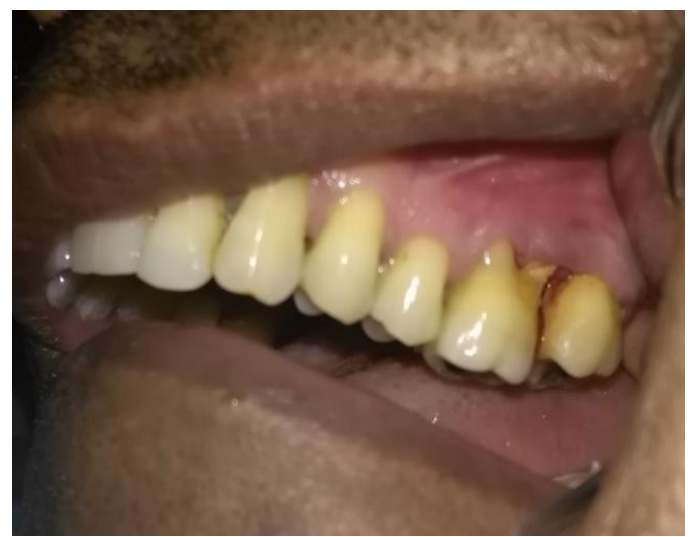

Fig.1: Pre- operative photograph

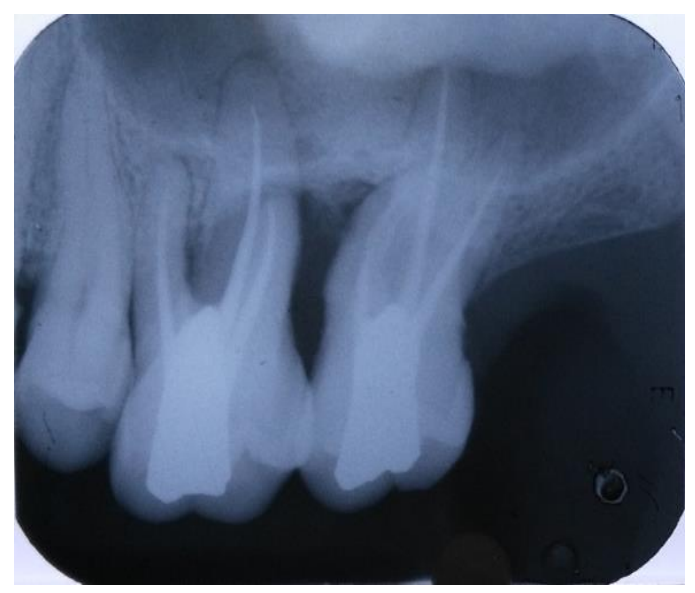

Fig. 2: Pre- operative radiograph

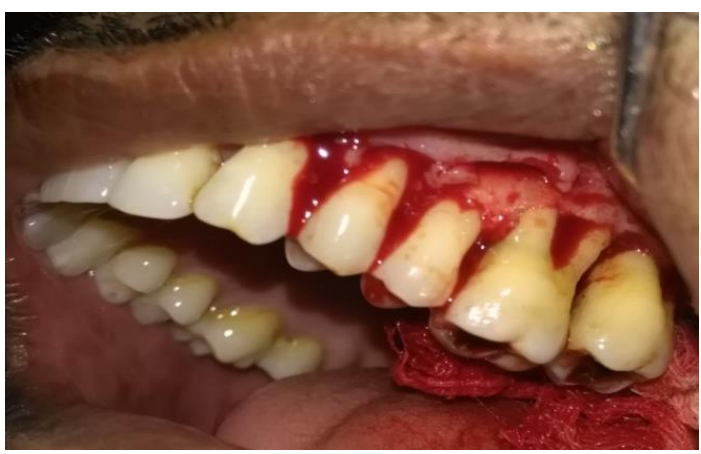

Fig. 3: After flap reflection

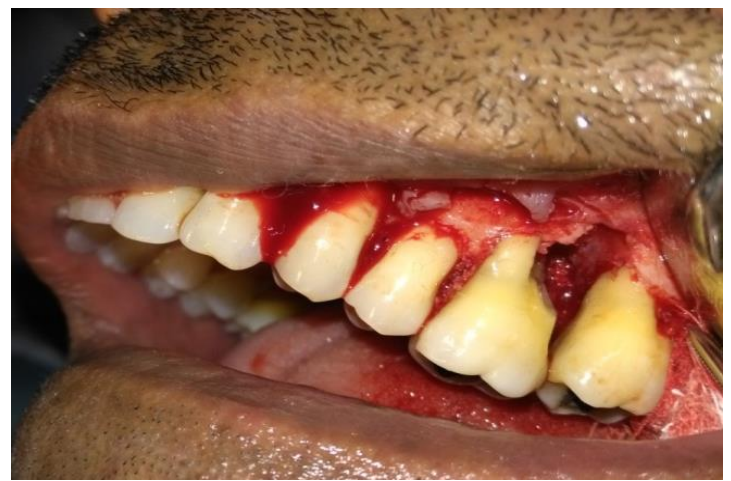

Fig. 4: Distobuccal root resection 


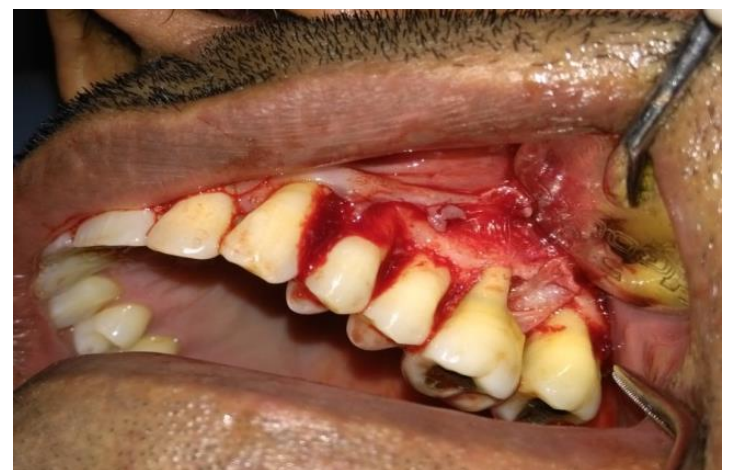

Fig. 5: Bone graft and PRF membrane placed

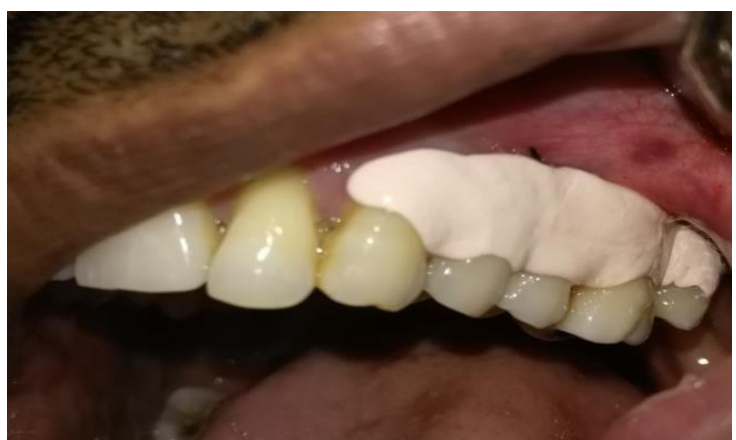

Fig. 6: COE pack given

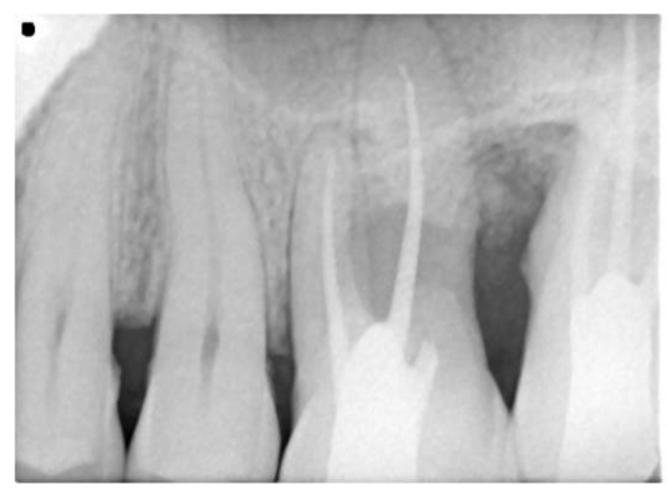

Fig. 7: One year post operative radiograph

\section{References}

1. Sumidha R, Pankaj B. Management of multirooted tooth with questionable prognosis through tooth resection: A Case Report. Heal Talk. 2010;9-12.

2. Amen C. Hemisection and root amputation. Periodontics. 1966;4:197.

3. DeSanctis M, Murphy KG.The role of resective periodontal surgery in the treatment of furcation defects. Periodontal. 2000;22:154-68.

4. Kamal N, Pooja M, Bharti S. Root Resection of Maxillary First Molar: A Case Report. IOSR-JDMS. eISSN: 2279-0853 p-ISSN: 2279-0861.Ver. IX 2017;16(4)103-7

5. Geeti G, Shruti C, Vipin A et al. The root resection of an endodontic periodontal lesion. Heal Talk. 2010;10-11.

6. Lindhe, J., Lang, N. P., \& Karring, T. Clinical periodontology and implant dentistry. Oxford: Blackwell Munksgaard. 2008

7. Mukhatar A, Safeena, Humera A. Root resection of maxillary first molar: A case report. Heal Talk.. 2012;4(4).

8. Marco Del Corso, Micheal Toffler, David M Dohan Ehrenfest. Use of autologous leukocyte and platelet rich fibrin (L-PRF) membrane in post avulsion sites a overview of Choukroun's PRF. The $j$ of implant and adv implant dent. 2010;1(9).

9. Newmann MG, Takei HH, Klokkevold PR, Carranza FA. Carranza's Clinical Periodontology. $10^{\text {th }} \mathrm{Ed}$. Philadelphia: W.B. Saunders Co.

How to cite this article: Chhabra P, Garg A, Chhabra P, Chhabra D. Root resection and PRF as an alternative treatment option: A case report. Int $\mathbf{J}$ Periodontol Implantol. 2018;3(4):128-130. 